\title{
On the Thermoelectricity of W. Thomson: Towards a Theory of Thermoelastic Conductors
}

\author{
J.D. Goddard
}

Received: 28 November 2010 / Published online: 22 January 2011

(C) The Author(s) 2011. This article is published with open access at Springerlink.com

\begin{abstract}
Thomson's classical analysis (1851, 1854a, 1854b) of thermoelectricity is cast into a modern form by means of contemporary thermodynamic methods based on the introduction of a free energy depending on strain, electrical charge and temperature. The present analysis identifies the phenomenological coefficients in Thomson's theory in terms of standard thermodynamic functions derivable from the free energy, lending support to the interpretation of his relations as authentic thermostatic relations. As an extension of Thomson's theory, the present work provides an analysis of the effect of tensile stress or pressure on thermoelectricity.

As a counterpoint to the analysis of Boltzmann (1877) and the representation of Onsager (1931), it is argued that the Onsager relations must be modified to include thermodynamically orthogonal forces and fluxes of the type identified by Ziegler (1970) and Edelen (1974). This allows for reversible coupling between the flow of heat and electricity postulated by Thomson and suggests the possibility of non-dissipative heat flux associated with microscopic thermoelectric effects.

The present analysis does not include a description of superficial charge concentration in conductors, which poses an interesting challenge for further analysis.
\end{abstract}

Keywords Thomson effect - Seebeck effect · Peltier effect · Volta effect . Piezo-thermoelectricity · Onsager relations · Thermodynamic orthogonality

Mathematics Subject Classification (2000) $80 \mathrm{~A}-02 \cdot 74 \mathrm{~A}-02$

\section{Introduction}

Thermoelectricity occupies a crucial position in the development of thermodynamics, dating from the from the earliest efforts to place electricity and mechanics on the same thermodynamic foundation by towering figures in the field [9, 32-34] and [1]. Thus, Thomson (later

J.D. Goddard $(\bowtie)$

Department of Mechanical and Aerospace Engineering, University of California, San Diego, 9500

Gilman Dr., La Jolla, CA 92093-0411, USA

e-mail: jgoddard@ucsd.edu 
Lord Kelvin) offers a thermostatic, i.e., equilibrium-thermodynamic, analysis of the Seebeck and Peltier effects, uncovering a new effect subsequently named after him. Thomson's stated reservations as to the provisional separation of these effects from the dissipative flow of electricity and heat were subsequently addressed by Boltzmann [1], in a comprehensive review of theory and experiment, followed by an analysis that leads him to conclude that such a separation is not justified. ${ }^{1}$

Both Thomson and Boltzmann analyze thermoelectricity as a thermodynamic system or device consisting of a thermocouple circuit, with Boltzmann concluding that the intimate connection between electrical and thermal conduction implies that such a circuit differs from a conventional (i.e., steam) heat-power cycle, for which an ever closer approach to reversibility can be achieved, at least in principle, by appropriate design of the process.

Moreover, in Boltzmann's analysis, as in that of Thomson, temperature and electrical potential are the sole intensive variables, as the notion of electrical free energy and the associated chemical potential had not yet been established in the literature. As a consequence, it is difficult to relate modern thermochemical concepts to the constitutive equations employed in those analyses, a matter which is addressed in the present work. An important exception is Thomson's "specific heat of electricity" [33], which clearly can be attributed to the thermochemical energy of electrons, later conceptualized and confirmed by experimental measurement [16, 21]. ${ }^{2}$

Bridgman [3] subsequently reviews Boltzmann's analysis and, while agreeing that Joule heat may mask reversible heat in a typical thermochemical circuit, concludes that this does not invalidate Thomson's formulae. In a later work, Bridgman [4] obtains the latter by a reworking of Thomson's analysis without, however, availing himself of the concept of electrochemical (Gibbs) free energy, by then well established in physical chemistry [20]. Following Bridgman's cogent physical reasoning, one may conclude that the routine application of the Seebeck effect to measure temperature is premised on its status as thermostatic potential. Once that premise is accepted, it follows logically that there must exist an associated reversible heat, completely analogous to the reversible heat associated with numerous thermodynamic phase transitions, and Thomson's relation connecting Seebeck and Peltier effects is tantamount to the well-known Clapeyron equation.

Despite the several important opinions in its favor, Thomson's theory was dealt a nearly fatal blow by the celebrated work of Onsager $[29]^{3}$ who treats thermoelectricity as strictly dissipative and subject to his now famous reciprocal relations. Following Onsager, numerous works subsequently indicate how Thomson's relations connecting Seebeck and Peltier effects can be interpreted according to Onsager's formalism [6, 19, 23]. However, none of

\footnotetext{
${ }^{1}$ Referring to Thompson's theory, he observes at the outset, "Die ... Theorie ist so schön in sich abgeschlossen, dass die Möglichkeit irher vollständig Übereinstimmung mit den Thatsachen nicht geleugnet werden kann." (The ... theory is so beautifully self-contained that the possibility of its complete consistency with the facts cannot be denied.)

${ }^{2}$ Modern theories of the solid state explain the fact that the electronic contribution to specific heat is generally much smaller than the contribution to thermal and electrical conduction (see e.g., [26]). Note that this and other citations of literature are selective and minimal, without attempt at a comprehensive or up-to-date review of thermoelectricity.

${ }^{3}$ Who attributes to Thomson a symmetric coupling of currents to potential gradients which I could not find explicitly formulated in the works cited by Onsager, [34] [journal title ostensibly mis-cited in [29]] and ([35], pp. 237-241), although one does find an appeal to symmetry in Thomson's description of the thermoelectric coupling in crystalline conductors ([35], I-pp. 266 ff.).
} 
these treatments seems to make a clear distinction between heat flowing within a conductor and heat exchange with the surroundings, either at the junction between conductors or along the length of a homogeneous conductor, a distinction I believe essential to a proper thermodynamic analysis.

The Boltzmann and Onsager points of view are now enshrined in several prominent treatises on thermodynamics and solid state physics, such as those of Sommerfeld ([31], pp. 157-162), ${ }^{4}$ Callen [7], Kittel [18], and Nye [28]. The book of Mott [26] represents a notable exception, but he does not relate Thomson's phenomenological coefficients to familiar thermodynamic functions. One goal of the present work is to do just that, as part of the effort to confirm the thermostatic origins and validity of Thomson's relations. As indicated above, the Seebeck-Peltier connection has a fairly evident thermostatic interpretation, whereas the status of the Thompson effect may be less clear. Therefore, an attempt is made below to define a static analogue of the latter.

In addition to the clarification of the above questions, a second goal of the present work is to lay down a foundation for a thermodynamic theory of stress-coupled thermoelectric effects (piezo-thermoelectricity), motivated largely by Thomson's pioneering experiments on the effect of tensile stress on metallic wires [34] and the subsequent works of Bridgman [2] and Hall [16] dealing with pressure effects on thermoelectricity. Some may find a more practical motivation in certain recently proposed device-technology based on coupled effects $[24,30]$.

The present work represents a fusion of classical thermoelasticity ${ }^{5}$ with thermoelectricity, allowing for the coupling of stress, heat flow and electrical flow. The analysis is modeled to some extent on well-known methods pioneered by Coleman [10]. While Coleman and Dill [11] briefly discuss the thermodynamics of electrical conductors, they relegate electrical current to the same status as dissipative heat flow, without considering electronic free energy and the associated thermoelectric coupling. By contrast, we shall draw on classical solution theory in a contemporary mixture-theoretical guise [15, 27], identifying electrons and electrical conductor as distinct constituents in a mixture, and thereby providing a theory that is readily extended to conducting media involving various ionic charge carriers (including the positive holes in semi-conductors).

In opposition to a considerable body of opinion dating from the above-cited work of Boltzmann, the current paper aims to resuscitate in modern form Thomson's notion of reversible coupling between heat and electrical flow. This coupling allows for non-dissipative currents not usually included in the standard Thermodynamics of Irreversible Processes as represented by the Onsager relations. As discussed in the Sect. 5, the underlying concept of thermodynamic orthogonality is a special case of that envisaged by Ziegler [36] and the Primitive Thermodynamics of Edelen [12]. Within this framework, one recovers Thomson's formula for thermoelectric power as a linear reversible term in electrical current with additive quadratic terms of the Onsager form.

\footnotetext{
${ }^{4}$ Who, after pursuing a development modeled on Thomson's approach, expresses the curious opinion, "There is little justification for such a separation of the so-called reversible and irreversible effects, in spite of the fact that it leads to a result that can be verified experimentally [emphasis added here]", citing the work of Boltzmann [1] as proof and then proceeding to an account of Onsager's theory without detailed application to thermo-electricity.

${ }^{5} \mathrm{An}$ area in which Professor Donald Carlson made several contributions, including a well-known comprehensive review of the classical theory [8].
} 


\section{Balance Equations and Thermodynamic Framework}

While the following analysis is based on the supposition of a smoothly varying chargedensity field inside our conductive body, we should recall the tendency of electrical conductors to concentrate charge on bounding surfaces, with charge density being zero almost everywhere in their interior. We could therefore adopt a different starting point, treating surface charge as a primary variable, with various consequences for the boundary conditions on other field variables, ${ }^{6}$ as done in other problems involving interfacial effects. ${ }^{7}$ Instead, we take the point of view that certain general conclusions drawn from an analysis based on balance laws for smoothly varying density fields should remain valid in the limit of fields with sharp gradients. Then, the major weak point in the resulting analysis lies in our adoption of constitutive equations for simple materials, i.e., materials depending only first spatial gradient of various potentials.

With specific charge ${ }^{8} q_{\mathrm{e}}=\varrho_{\mathrm{e}} / \rho$ and total flux, i.e. current density $\boldsymbol{\varphi}_{\mathrm{e}}=\mathbf{j}_{\mathrm{e}}+\varrho_{\mathrm{e}} \mathbf{v}$, where $\mathbf{v}$ is barycentric velocity, then $\mathbf{j}_{\mathrm{e}}$ represents relative current density, and the conservation of charge has the respective spatial and material forms:

$$
\partial_{t} \varrho_{\mathrm{e}}+\nabla \cdot \varphi_{\mathrm{e}}=\rho \dot{q}_{\mathrm{e}}+\nabla \cdot \mathbf{j}_{\mathrm{e}}=0
$$

The mass balance is of course given by writing $\rho$ for $\varrho_{\mathrm{e}}$ and $\rho \mathbf{v}$ for $\boldsymbol{\varphi}_{\mathrm{e}}$.

As done by numerous investigators (e.g. [22]), we could identify free (f) and bound (b) charge, with $\mathfrak{a}_{\mathrm{e}}=\mathfrak{a}_{\mathrm{f}}+\mathfrak{a}_{\mathrm{b}}$, for $\mathfrak{a}=\varrho, \boldsymbol{\varphi}, \mathbf{j}$, subject to (1). Anticipating certain aspects of semiconductor physics, Hall [16] allows for interconversion between the two, with materially bound charge designated as "associated". In like manner we could consider an ionic system with any number of charged species $i=1,2, \ldots$, with $\mathfrak{a}_{i}$ involving integral multiples of electronic charge, ${ }^{9}$ but the focus here is on electrons as the sole charge carriers.

Account taken of electronic effects, we adopt as internal energy balance:

$$
\rho \dot{\epsilon}=\operatorname{tr}(\mathbf{T L})-\nabla \cdot \mathbf{q}-\nabla \cdot\left(h_{\mathrm{e}} \mathbf{j}_{\mathrm{e}}\right)-\mathbf{j}_{\mathrm{e}} \cdot \nabla \Phi+\rho r,
$$

where $\mathbf{T}$ denotes the stress tensor, $\mathbf{L}:=(\nabla \mathbf{v})^{\mathrm{T}}$ velocity gradient, $\epsilon$ specific internal energy, q conductive heat flux, $\Phi$ external electrical potential, $\rho r$ radiant energy supply, and $h_{\mathrm{e}}$ specific electronic enthalpy. The latter is related to electronic entropy $\eta_{\mathrm{e}}$ and chemical potential (Gibbs free energy) $\mu_{\mathrm{e}}$ by

$$
h_{\mathrm{e}}=\mu_{\mathrm{e}}+\theta \eta_{\mathrm{e}},
$$

with absolute temperature $\theta$ assumed to be the same for electrons and conductor. Based on specific constitutive assumptions, these definitions will be made more precise below.

\footnotetext{
${ }^{6}$ Equivalently, one might express charge densities as distributions (generalized functions), representing the dual of conjugate fields in virtual-work principles.

${ }^{7}$ Burak and Galapats [5] cite numerous references to the Russian literature on ionic conduction and discuss the general importance of surface charge, of the type discussed by Bridgman [3] for conductors and by Kittel [18], p. 551, for semi-conductors.

${ }^{8}$ Here and in the following, charge is taken as intrinsically positive and expressed as multiples of the Faraday constant $\mathcal{F}=\mathcal{N} Q_{\mathrm{e}}$, where $Q_{\mathrm{e}}$ denotes charge of the electron (treated as positive) and $\mathcal{N}$ Avogadro's number.

${ }^{9}$ In the usual theories of ionic systems, the charge density of species $i$ is given by $\varrho_{i}=\hat{q}_{i} \rho_{i}$, where $\hat{q}_{i}$ is charge/mass ratio and $\rho_{i}$ mass density, with some authors treating conduction electrons in this fashion (e.g., [16]).
} 
The balance (2) differs from that proposed in other investigators, such as the classical approach of Sommerfeld [31] and the more modern work of Gurtin and Vargas [15], who employ a term $\nabla \cdot\left(\mu_{\mathrm{e}} \mathbf{j}_{\mathrm{e}}\right)$ instead of $\nabla \cdot\left(h_{\mathrm{e}} \mathbf{j}_{\mathrm{e}}\right)$, implying a different definition of the heat flux or mechanical work in (2). For example, by redefining heat flux as $\mathbf{q}+h_{\mathrm{e}} \mathbf{j}_{\mathrm{e}}$ the present energy and entropy balances reduce to those of Lebon et al. [19] for the case of no stress work. It is plausible that the simple-material model employed here will always allow a certain latitude in such definitions (cf. [14]).

In formulating the entropy balance, we distinguish conductive heat flow qua diffusive energy flux from diffusive entropy flux, as done e.g. by Müller [27] in a more general setting, writing

$$
\rho \dot{\eta} \geq-\nabla \cdot \mathbf{j}_{S}+\frac{\rho r}{\theta}, \quad \text { where } \mathbf{j}_{S}=\frac{\mathbf{q}}{\theta}+\eta_{\mathrm{e}} \mathbf{j}_{\mathrm{e}}
$$

where $\eta$ denotes specific entropy. Then, the combined energy-entropy or free-energy balance can be written:

$$
\begin{aligned}
\rho \dot{\psi} & \leq-\rho \eta \dot{\theta}+\operatorname{tr}(\mathbf{T L})-\mathbf{j}_{S} \cdot \nabla \theta-\nabla \cdot\left(\phi_{\mathrm{e}} \mathbf{j}_{\mathrm{e}}\right) \\
& =-\rho \eta \dot{\theta}+\operatorname{tr}(\mathbf{T L})-\mathbf{j}_{S} \cdot \nabla \theta-\mathbf{j}_{\mathrm{e}} \cdot \nabla \phi_{\mathrm{e}}+\rho \dot{q}_{\mathrm{e}} \mu_{\mathrm{e}},
\end{aligned}
$$

where

$$
\psi=\epsilon-\theta \eta, \quad \text { and } \quad \phi_{\mathrm{e}}=\mu_{\mathrm{e}}+\Phi,
$$

representing, respectively, (Helmholtz) free energy and total electromotive potential. ${ }^{10}$ The relation (5), with $\Phi \equiv 0, q_{\mathrm{e}} \equiv 0$, is equivalent to the balance of Gurtin and Vargas [15], for a mixture with two constituents and with no chemical reaction, provided their $\mathbf{q}$ is replaced by $\mathbf{j}_{S}$.

\subsection{Elastic Conductors}

We adopt as constitutive theory, an elastic frame-indifferent simple material, such that

$$
\psi=\psi\left(\mathbf{U}, \theta, q_{\mathrm{e}}, \nabla \theta, \nabla q_{\mathrm{e}}\right), \quad \text { where } \mathbf{U}=\sqrt{\mathbf{F}^{\mathrm{T}} \mathbf{F}}, \mathbf{F}=\partial \mathbf{x} / \partial \mathbf{x}^{\mathrm{o}},
$$

$\mathbf{F}$ denoting the gradient of the deformation, $\mathbf{x}^{\mathrm{o}} \rightarrow \mathbf{x}$, and $\mathbf{U}$ its right stretch. Then, wellknown arguments based on (5), e.g., of Gurtin and Vargas [15] in their extension of the theory of Coleman [10], lead to the simpler constitutive equation $\psi=\psi\left(\mathbf{U}, \theta, q_{\mathrm{e}}\right)$.

With the decomposition $\mathbf{U}=\mathbf{U}_{1} \mathbf{U}_{2}$ into isochoric shear $\mathbf{U}_{1}=\mathbf{S}=\left(\rho / \rho^{\mathrm{o}}\right)^{1 / 3} \mathbf{U}$ and isotropic expansion $\mathbf{U}_{2}=\left(\rho^{\mathrm{o}} / \rho\right)^{1 / 3} \mathbf{I}$, with $\psi=\psi\left(\mathbf{S}, v, \theta, q_{\mathrm{e}}\right)$, the relation (5) gives

$$
\partial_{\mathbf{S}} \psi=(\rho \mathbf{S})^{-1} \mathbf{T}^{\prime}, \quad \partial_{v} \psi=-p, \quad \partial_{\theta} \psi=-\eta, \quad \partial_{\mathrm{e}} \psi=\mu_{\mathrm{e}}, \quad \text { where } \partial_{\mathrm{e}}=\frac{\partial}{\partial q_{\mathrm{e}}},
$$

where $\mathbf{T}^{\prime}$ is deviatoric stress and $v=\rho^{-1}$ is specific volume. We recall that the Gibbs freeenergy, representing the Legendre conjugate $\mu=\mu\left(\mathbf{S}, p, \theta, q_{\mathrm{e}}\right)=\psi+p v$, satisfies

$$
\partial_{\mathbf{S}} \mu=(\rho \mathbf{S})^{-1} \mathbf{T}^{\prime}, \quad \partial_{p} \mu=v, \quad \partial_{\theta} \mu=-\eta, \quad \partial_{\mathrm{e}} \mu=\mu_{\mathrm{e}}
$$

\footnotetext{
${ }^{10}$ With $q_{\mathrm{e}} \phi_{\mathrm{e}}$ representing the electrochemical potential in ionic systems, as summarized at http://en.wikipedia.org/wiki/Electrochemical_potential.
} 
with appropriate re-interpretation of partial derivatives. From this, one obtains the useful (Maxwell) relation for isobaric or isochoric processes, respectively,

$$
\left(\partial \mu_{\mathrm{e}} / \partial \theta\right)_{\mathbf{S}, z, q_{\mathrm{e}}}=-\eta_{\mathrm{e}}, \quad \text { with } z=p \text { or } v .
$$

All thermostatic equations of state are determined by specification of $\psi\left(\mathbf{S}, v, \theta, q_{\mathrm{e}}\right)$, and such relations simplify in the case of thermally ideal conductors, where

$$
\psi\left(\mathbf{S}, v, \theta, q_{\mathrm{e}}\right)=q_{\mathrm{e}} \psi_{\mathrm{e}}(\mathbf{S}, v, \theta)+\psi_{0}(\mathbf{S}, v, \theta),
$$

the analogue of ideal gases mixtures and ideal solutions, for which a sum over e is involved. A similar relation then applies to $\mu\left(\mathbf{S}, p, \theta, q_{\mathrm{e}}\right)$. Whereas several treatments of thermoelectricity are based on (11), non-idealities arising from ionic interactions in semi-conductors have also been addressed, e.g., by means of the well-known Debye-Hückel theory. While contemporary theories of the solid state also relate electronic free energy to the Fermi levels in semi-conductors $[18,26,28]$, our thermodynamic analysis presumably does not depend on such details, as interesting as they may be for solid-state physics and material science.

\subsubsection{Dissipation Inequalities}

In addition to (7)-(9), the relation (5) gives the dissipation as

$$
\mathcal{D}:=-\mathbf{j}_{S} \cdot \nabla \theta-\mathbf{j}_{\mathrm{e}} \cdot \nabla \phi_{\mathrm{e}} \geq 0
$$

For processes in which only temperature varies, (10) and (12) give the alternative form

$$
\mathcal{D}=-\mathbf{q} \cdot \nabla \ln \theta-\mathbf{j}_{\mathrm{e}} \cdot \nabla \Phi \geq 0
$$

In the formalism of the linear Thermodynamics of Irreversible Processes [19, 22], (12) assumes an abstract six-dimensional vector format, with

$$
\underline{X}=-\left\{\nabla \phi_{\mathrm{e}}, \nabla \theta\right\}, \quad \underline{J}=\left\{\mathbf{j}_{\mathrm{e}}, \mathbf{j}_{S}\right\}, \quad \text { with } \mathcal{D}=\underline{X}^{\mathrm{T}} \underline{J} \geq 0
$$

and with a similar expression for (13). We recall that this is the starting point of the treatment of thermoelectricity by Onsager [29], a matter discussed further below. We first show that the relations (7)-(14) serve to reproduce Thomson's analysis of strictly reversible thermoelectric effects.

In the following quasi-static analysis we do not deal explicitly with spatial variations in $\mathbf{S}, q_{\mathrm{e}}$ and $v$ or $p$, assuming that minimization of global free energy

$$
\int_{V} \psi\left(\mathbf{S}, v, \theta, q_{\mathrm{e}}\right) d V(\mathbf{x}) \quad \text { or } \quad \int_{V} \mu\left(\mathbf{S}, p, \theta, q_{\mathrm{e}}\right) d V(\mathbf{x})
$$

subject to appropriate constraints, would determine equilibrium fields $\mathbf{S}, q_{\mathrm{e}}$ and $v$ or $p$, given $\theta$. Hence, all these quantities are allowed to vary with changes in $\theta$, subject to the same constraints, and derivatives $\partial_{\theta}$ are to be interpreted accordingly. 


\section{Non-dissipative Steady States}

Non-dissipative states or processes are those for which $\mathcal{D}=0$ in (12)-(14), representing thermodynamic-equilibrium (thermostatic) states and reversible processes, as illustrated by the following examples involving quasi-static, isobaric and constant-stretch processes.

With proper integral balances, we could identify external heat exchange with the normal component of $\mathbf{q}$ on appropriately defined boundaries. However, for the sake of convenience, we let the nominal radiation $\rho r$ appearing in the balances (2)-(4) represent a general heat exchange with the surroundings.

\subsection{Adiabatic Flow}

For $\rho r \equiv 0$, the steady-state entropy balance (4) reduces to $\nabla \cdot\left(\mathbf{q} / \theta+\eta_{\mathrm{e}}\right)=0$, whose solution is

$$
\mathbf{q}+\theta \eta_{\mathrm{e}} \mathbf{j}_{\mathrm{e}}=-\theta \mathbf{b}(\mathbf{x}), \quad \text { where } \nabla \cdot \mathbf{b}=0 .
$$

Then, for the conditions at hand, the energy balance (2) has the solution

$$
\phi_{\mathrm{e}} \mathbf{j}_{\mathrm{e}}=\mathbf{a}+\theta \mathbf{b}, \quad \text { where } \nabla \cdot \mathbf{a}=0 .
$$

For the one-dimensional case, representing flow along an idealized insulated wire, vectors reduce to scalar functions of distance $x$ along the wire, with $\mathbf{a}$ and $\mathbf{b}$ reducing to scalar constants $a, b$. Then (16) represents one possible reversible transition between terminals at different potential and temperature, and with appropriate choice of the constants $a, b$ (16) can be written

$$
\begin{aligned}
& \phi_{\mathrm{e}}(x) j_{\mathrm{e}}=\left(1-\frac{\theta}{\theta_{\mathrm{H}}}\right) q_{\mathrm{H}}+\left(\Phi_{\mathrm{H}}+h_{\mathrm{eH}}-\theta \eta_{\mathrm{eH}}\right) j_{\mathrm{e}}, \quad \text { so that } \\
& {\left[\Phi_{\mathrm{H}}-\Phi_{\mathrm{C}}+h_{\mathrm{eH}}-h_{\mathrm{eC}}-\theta_{\mathrm{C}}\left(\eta_{\mathrm{eH}}-\eta_{\mathrm{eC}}\right)\right] j_{\mathrm{e}}=\left(1-\frac{\theta_{\mathrm{C}}}{\theta_{\mathrm{H}}}\right) q_{\mathrm{H}},}
\end{aligned}
$$

where $q_{\mathrm{H}}$ is the heat flow at the nominal high temperature terminal, and the quantity in square brackets represents electrochemical exergy, relative to the nominal low temperature terminal. While not recorded here, one can work out an analogous result for a threedimensional conductor with suitable boundary conditions.

\subsection{Thermally-Isentropic Flow}

We consider the case

$$
\nabla \cdot\left(\frac{\mathbf{q}}{\theta}\right)=0, \quad \therefore \quad \mathbf{q}=-\mathbf{b}(\mathbf{x}) \theta, \quad \text { with } \nabla \cdot \mathbf{b}(\mathbf{x})=0, \quad \text { and } \quad \rho r=\theta \mathbf{j}_{\mathrm{e}} \cdot \nabla \eta_{\mathrm{e}} .
$$

The last relation follows from (4) and represents the Thomson effect discussed below. In view of the electrical entropy transport in (4), the designation "thermally-isentropic" seems more appropriate than "isentropic".

The following variant on (16) follows from (2):

$$
\Phi \mathbf{j}_{\mathrm{e}}=\mathbf{a}(\mathbf{x})-\mathbf{q} \equiv \mathbf{a}(\mathbf{x})+\theta \mathbf{b}(\mathbf{x}), \quad \nabla \cdot \mathbf{a}(\mathbf{x})=0,
$$

where the functions $\mathbf{a}, \mathbf{b}$ are generally different from those appearing in (16). 
In the case of a one-dimensional conductor, with one end at $\theta=\theta_{\mathrm{H}}$ and the other at $\theta=\theta_{\mathrm{C}} \leq \theta_{\mathrm{H}}$, (19) gives the (Carnot-Thomson) relation:

$$
j_{\mathrm{e}}\left(\Phi_{\mathrm{C}}-\Phi_{\mathrm{H}}\right)=q_{\mathrm{H}}-q_{\mathrm{C}}=q_{\mathrm{H}}\left(1-\frac{\theta_{\mathrm{C}}}{\theta_{\mathrm{H}}}\right)=q_{\mathrm{C}}\left(\frac{\theta_{\mathrm{H}}}{\theta_{\mathrm{C}}}-1\right)
$$

representing "heat-engine" or "heat-pump" according to the algebraic sign of $q_{\mathrm{H}}$.

It should be noted that (20) does not contain the terms in $\mu_{\mathrm{e}}$ appearing in (16)-(17), since such terms are balanced by external heat exchange. Furthermore, as a global balance, (20) tells us nothing about the magnitudes of heat effect or potential difference, a matter requiring constitutive relations.

\subsubsection{The Thomson Effect}

This is usually defined as reversible heat exchange of between a conductor and its surroundings accompanying steady current flow. We note that the effect is not necessarily relevant to the thermocouple circuit discussed below, which may for example involve a reversible adiabatic transition between contacts.

To obtain Thomson's formulae within the present thermodynamic framework, we assume a thermally isentropic flow described by (18), whose last member gives the heat exchange in question as:

$$
\rho r=\theta \nabla \cdot \eta_{\mathrm{e}} \mathbf{j}_{\mathrm{e}}=\mathcal{T} \mathbf{j}_{\mathrm{e}} \cdot \nabla \theta, \quad \text { where } \mathcal{T}=\frac{1}{\theta} \partial_{\theta} \eta_{\mathrm{e}} \equiv \partial_{\theta} h_{\mathrm{e}}=c_{p \mathrm{e}}
$$

Therefore, the Thomson coefficient $\mathcal{T}$, which is usually denoted by the symbol $\mu$ and almost always associated with one-dimensional flow, is identical with the isobaric specific heat $c_{p e}$ (Thomson's specific heat of electricity). ${ }^{11}$

It is clear that the Thomson effect requires a spatially variable ambient temperature, if all external heat transfer is to take place at the spatially variable temperature of the conductor. Perhaps more important is the fact that the effect, by its very definition, involves a temperature gradient in the conductor leading inevitably to dissipative effects from heat flow. Of course, neither fact invalidates the thermostatic interpretation of (21).

\subsection{Iso-exergic Flow}

With electrochemical potential designated as (absolute) exergy, and with $\mathcal{D}=0$ in (12) an iso-exergic flow or process is defined by:

$$
\phi_{\mathrm{e}}=\mu_{\mathrm{e}}+\Phi=\text { const., } \quad \text { with } \quad \therefore \quad \mathbf{j}_{S}=\mathbf{q} / \theta+\eta_{\mathrm{e}} \mathbf{j}_{\mathrm{e}}=\mathbf{0} \text {, or } \theta=\text { const. }
$$

representing either isentropic flow, in which heat flux cancels electrical entropy transport, or isothermal flow. Either case involves reversible heat exchange with the surroundings and both are relevant to the prominent thermoelectric effects discussed next.

\footnotetext{
${ }^{11} \mathrm{Cf}$. [33]. In an appropriate system of units, his symbols $J, F, t, \mu, \Theta, \vartheta$ translate, respectively, to 1 , $V^{\mathrm{AB}}, \theta, 1 / \theta,-\Pi, c_{p \mathrm{e}}$ in the present notation. His "vitreous" and "resinous" electricity correspond to the modern concept of positive and negative charge.
} 


\subsubsection{Seebeck \& Peltier Effects and Thomson's Formula}

While the case of isothermal flow is relatively uninteresting for homogeneous materials, passage of electrical current through the junction between heterogeneous conductors, i.e. a heterojunction, generally involves a jump in electrical potential, or thermocouple junction emf, at the interface or junction between materials $\mathrm{A}, \mathrm{B}$, where $\phi_{\mathrm{e}}$ is presumably continuous. ${ }^{12}$ The emf is then given by the first member of (22) by the increment of chemical potential as

$$
\Phi^{\mathrm{AB}}=-\mu_{\mathrm{e}}^{\mathrm{AB}}, \quad \text { where }()^{\mathrm{AB}}:=()^{\mathrm{A}}-()^{\mathrm{B}},
$$

where $\mu_{\mathrm{e}}^{\mathrm{I}}=\mu_{\mathrm{e}}^{\mathrm{I}}\left(\mathbf{S}^{\mathrm{I}}, v^{\mathrm{I}}, \theta,, q_{\mathrm{e}}^{I}\right)$ for $\mathrm{I}=\mathrm{A}, \mathrm{B}$. The temperature dependence and associated heat effect underlie the most prominent thermoelectric phenomena.

The Seebeck (or thermocouple) effect refers to the change with temperature of the junction potential (23), with Seebeck coefficient given by

$$
\mathcal{S}^{\mathrm{AB}}:=\partial_{\theta} \Phi^{\mathrm{AB}}=-\partial_{\theta} \mu_{\mathrm{e}}^{\mathrm{AB}}=\eta_{e}^{\mathrm{AB}} .
$$

This renders transparent a standard formula connecting Thomson and Seebeck effects, as represented by the first and last identities in the expression: ${ }^{13}$

$$
\mathcal{S} \equiv \eta_{\mathrm{e}}(\theta)=\int_{0}^{\theta} c_{p \mathrm{e}}\left(\theta^{\prime}\right) d \ln \theta^{\prime} \equiv \int_{0}^{\theta} \mathcal{T}\left(\theta^{\prime}\right) d \ln \theta^{\prime},
$$

where the integrals, taken along a path of constant $\mathbf{S}, p, q_{\mathrm{e}}$, are assumed to converge at the lower limit, and where we have suppressed notation for dependence on $\mathbf{S}, p, q_{\mathrm{e}}$.

The Peltier effect refers to reversible external heat exchange at a junction or interface, given by the discontinuity in normal heat flux. Thus, the second relation in (22) serves to define the Peltier coefficient $\Pi^{\mathrm{AB}}$ and to establish its relation to the Seebeck coefficient:

$$
\mathbf{n} \cdot \mathbf{q}^{\mathrm{AB}}=-\Pi^{\mathrm{AB}} \mathbf{n} \cdot \mathbf{j}_{\mathrm{e}}, \quad \text { with } \Pi^{\mathrm{AB}}=\theta \eta_{\mathrm{e}}^{\mathrm{AB}} \equiv \theta \mathcal{S}^{\mathrm{AB}} .
$$

By means of the preceding relations it is a relatively easy matter to derive Thomson's formula for the open-circuit electromotive potential (thermocouple emf) of a circuit consisting of two different metals A, B with junctions at different temperatures. Thus, for his example ([33], Sect. II, p. 53) of current flow from A to B through the hotter junction at $\theta_{H} \geq \theta_{C}$ and flow from B to A through the junction at $\theta_{\mathrm{C}}$, with $\Phi$ constant in each conductor, or else with cancellation of potential drops in the adiabatic flow in each conductor, the desired quantity is obtained from the first condition in (22) as the sum of junction potentials:

$$
V^{\mathrm{AB}}\left(\theta_{\mathrm{H}}, \theta_{\mathrm{C}}\right)=\Phi^{\mathrm{AB}}\left(\theta_{\mathrm{H}}\right)+\Phi^{\mathrm{BA}}\left(\theta_{\mathrm{C}}\right)=\left[\mu_{\mathrm{e}}^{\mathrm{B}}\left(\theta_{\mathrm{H}}\right)-\mu_{\mathrm{e}}^{\mathrm{B}}\left(\theta_{\mathrm{C}}\right)\right]-\left[\mu_{\mathrm{e}}^{\mathrm{A}}\left(\theta_{\mathrm{H}}\right)-\mu_{\mathrm{e}}^{\mathrm{A}}\left(\theta_{\mathrm{C}}\right)\right] .
$$

Then, making use of the relations:

$$
\begin{aligned}
\mu_{\mathrm{e}}\left(\theta_{\mathrm{H}}\right)-\mu_{\mathrm{e}}\left(\theta_{\mathrm{C}}\right) & =h_{\mathrm{e}}\left(\theta_{\mathrm{H}}\right)-h_{\mathrm{e}}\left(\theta_{\mathrm{C}}\right)-\left[\theta_{\mathrm{H}} \eta_{\mathrm{e}}\left(\theta_{\mathrm{H}}\right)-\theta_{\mathrm{C}} \eta_{\mathrm{e}}\left(\theta_{\mathrm{C}}\right)\right] \\
& =\int_{\theta_{\mathrm{C}}}^{\theta_{\mathrm{H}}} c_{p \mathrm{e}}(\theta) d \theta-\theta_{\mathrm{H}} \int_{\theta_{\mathrm{C}}}^{\theta_{\mathrm{H}}} c_{p \mathrm{e}}(\theta) d \ln \theta+\theta_{\mathrm{C}} \int_{\theta_{\mathrm{C}}}^{\theta_{\mathrm{H}}} c_{p \mathrm{e}}(\theta) d \ln \theta,
\end{aligned}
$$

\footnotetext{
${ }^{12}$ We recall that continuity of $\mathbf{j}_{\mathrm{e}}$ under quasi-static conditions, where there exists no transient accumulation of bulk or surface charge, insures continuity of $\phi_{\mathrm{e}}$, by familiar virtual-work principles.

${ }^{13}$ With $\mathcal{S}$ usually denoted by the infelicitous term "thermoelectric power" (cf. [26], pp. 308-310).
} 
together with (24)-(26) and a bit of algebra, one finds the formula of Thomson [33]:

$$
V^{\mathrm{AB}}\left(\theta_{\mathrm{H}}, \theta_{\mathrm{C}}\right)=\Pi^{\mathrm{AB}}\left(1-\frac{\theta_{\mathrm{C}}}{\theta_{\mathrm{H}}}\right)+\int_{\theta_{\mathrm{C}}}^{\theta_{\mathrm{H}}} c_{p \mathrm{e}}(\theta)\left(1-\frac{\theta_{\mathrm{C}}}{\theta}\right) d \theta
$$

which he obtains by a tour-de-force application of Carnot's Principle. ${ }^{14}$

\subsubsection{A Static Thomson Effect}

As further support for Thomson's results, one can envisage a static version of his effect involving the emf between two conductors $\mathrm{H}, \mathrm{C}$ of the same material at different temperatures, which appears identical with the thermal Volta effect postulated by Bridgman [3].

Thus, we assume each conductor to be in contact with its own isobaric heat bath, at respective temperatures $\theta_{\mathrm{H}}, \theta_{\mathrm{C}} \leq \theta_{\mathrm{H}}$, with each conductor and its heat bath insulated thermally from the other. If the conductors are then connected electrically to one another by a thermally insulating capacitor (e.g., parallel conducting plates in vacuo) of negligible thermal capacity, one can envisage the possibility of a quasi-static transfer of charge $Q_{\mathrm{e}}$ from Conductor $\mathrm{H}$ to the capacitor, with equal and opposite transfer from Conductor $\mathrm{C}$. Then, the global entropy balance and free-energy balances give

$$
\begin{aligned}
& Q_{\mathrm{N}}=Q_{\mathrm{H}}-Q_{\mathrm{C}}=Q_{\mathrm{H}}\left(1-\frac{\theta_{\mathrm{C}}}{\theta_{\mathrm{H}}}\right)=\int_{0}^{Q_{\mathrm{e}}}\left(\Phi_{\mathrm{H}}-\Phi_{\mathrm{C}}\right) d Q_{\mathrm{e}}, \quad \text { and } \\
& Q_{\mathrm{N}}=Q_{\mathrm{e}} \int_{\theta_{\mathrm{C}}}^{\theta_{\mathrm{H}}} c_{p \mathrm{e}}(\theta) d \theta,
\end{aligned}
$$

where $Q_{\mathrm{H}}, Q_{\mathrm{C}}$ denote heat transferred to and from conductors, respectively, with net transfer $Q_{\mathrm{N}}$. The integral in the first relation represents electrostatic energy of the capacitor, and the derivative $\partial\left(Q_{\mathrm{N}} / Q_{\mathrm{e}}\right) / \partial \theta_{\mathrm{H}}$, represents the static Thomson effect. As with the dynamic version, the Carnot-cycle work $Q_{\mathrm{H}}\left(1-\theta_{\mathrm{C}} / \theta_{\mathrm{H}}\right)$ is converted to electrical potential energy, and an equal amount of heat is converted into electronic enthalpy.

\section{Stress Effects}

Thomson [33] gives a brief summary of his experiments on the effect of stress on the thermoelectricity, as indicated by the appearance of a junction potential between stretched and unstretched copper wires, while Hall [16, 17] and Bridgman [2] study the effects of isotropic pressure.

Although Thomson's theoretical work on crystalline conductors [34] portends a more modern and comprehensive theory of conduction in elastic bodies, we focus here on the illustrative example of an idealized one-dimensional conductor as a model of his experiment on metallic wires. Thus, with stretch ratio $\lambda=\partial x / \partial x^{0}$ replacing the right stretch $\mathbf{U}$ as the sole kinematic variable, we obtain the reduced constitutive equations

$$
\gamma=\ln \lambda, \quad \psi=\psi\left(\gamma, \theta, q_{\mathrm{e}}\right), \quad \tau=\partial_{\gamma} \psi, \quad \mu=\mu\left(\tau, \theta, q_{\mathrm{e}}\right):=\psi-\tau \gamma
$$

\footnotetext{
${ }^{14}$ By making use of a differential version of (20), with $d q=c_{p \mathrm{e}} d T$ on the paths between heterojunctions, together with (26) for those junctions.
} 
Here $\gamma$ denotes Hencky strain and $\tau$ its work-conjugate tension, with

$$
\tau_{\mathrm{e}}=\partial_{\mathrm{e}} \tau \quad \text { and } \quad \gamma_{\mathrm{e}}=\partial_{\mathrm{e}} \mu
$$

These are analogues of the pressure-volume formulation envisaged by Hall [16], with $\tau=$ $-p, \gamma=v$, and with $p_{\mathrm{e}}=-\tau_{\mathrm{e}}$ representing his electronic (partial) pressure. We can now establish certain connections between various piezo-thermoelectric effects.

First, note that the junction potential between the same material in different states of mechanical tension is

$$
\Phi^{\mathrm{AB}}=-\mu_{\mathrm{e}}(\tau, \theta)+\mu_{\mathrm{e}}\left(\tau^{\mathrm{o}}, \theta\right), \quad \text { with } \tau^{\mathrm{A}}=\tau, \tau^{\mathrm{B}}=\tau^{\mathrm{o}},
$$

where, for reasons stated above, we suppress notation for dependence on the $q_{\mathrm{e}}$, assuming them to be determined by $\tau, \tau^{\circ}$ and $\theta$. Hence, (26), (24) and (32) leads to the relations

$$
\partial_{\tau} \mathcal{S}^{\mathrm{AB}}=\frac{1}{\theta} \partial_{\tau} \Pi^{\mathrm{AB}}=\int_{0}^{\theta} \partial_{\tau} \mathcal{T}^{\mathrm{AB}} d \ln \theta
$$

connecting stress response of Seebeck, Peltier and Thomson effects. Not only do these relations allow for a theoretical interpretation of thermo-electric experiments on stretched conductors, but they also may have application to devices such as temperature-sensitive strain gauges for the investigation of thermoelastic phenomena.

\section{Thermodynamic Orthogonality and the Modification of Onsager's Relations}

Based on his view of thermo-electricity as irreversible, [29] represents the linear theory by means of his phenomenological relations for forces and fluxes, such as those in (14), by

$$
\underline{X}=\mathfrak{R} \underline{J}, \quad \text { or } \quad \underline{J}=\mathfrak{L} \underline{X}, \quad \text { with } \mathfrak{L}=\mathfrak{R}^{-1},
$$

where $\mathfrak{L}$ and $\mathfrak{R}$ are positive-definite symmetric matrices, such that dissipation $\mathcal{D}$ is given by the (Rayleigh) form:

$$
\mathcal{D}=\underline{X}^{*} \underline{J}=\underline{J}^{*} \mathfrak{R} \underline{J}=\underline{X}^{*} \mathfrak{L} \underline{X}>0, \quad \text { for }|\underline{J}|=\sqrt{\underline{J}^{*} \underline{J}} \neq 0 .
$$

Here as below, the superscripts * denote elements of an appropriate dual space and will generally involve a suitable metric. (By suitable choice of variables $\underline{X}, \underline{J}$, duals can be represented by abstract vector transposes.)

As an extension of (34), we add a non-dissipative force, writing

$$
\underline{X}=\underline{X}_{\mathrm{eq}}+\mathfrak{R} \underline{J}, \quad \text { with } \underline{X}_{\mathrm{eq}}=\mathfrak{N} \underline{\hat{J}}+\underline{Y}-\left(\underline{\hat{J}}^{*} \underline{Y}\right) \underline{\hat{J}} \text {, where } \underline{\hat{J}}=\frac{\underline{J}}{|\underline{J}|}, \mathfrak{N}^{*}=-\mathfrak{N} \text {, }
$$

where the arbitrary vector $\underline{Y}$ and $\mathfrak{N}$ may both depend on $\underline{\hat{J}}$. Then, the rate-independent force $\underline{X}_{\text {eq }}$ obviously lies in the subspace normal to $\underline{J}$, makes no contribution to dissipation $\mathcal{D}$, and represents one form of the thermodynamic orthogonality of Ziegler [36] and Edelen [12].

If one exchanges the roles of force $\underline{X}$ and flux $\underline{J}$ in Edelen's Primitive Thermodynamics, one obtains a nonlinear resistance generalization of (36) on replacing the term $\mathfrak{R}$ by the 
gradient $\partial_{\underline{J}} \phi$ of a dissipation potential $\phi(\underline{J})$, with $\underline{X}_{\text {eq }}$ playing the role of a work-free force ${ }^{15}$ (in lieu of Edelen's "powerless" flux).

For the present purposes, we consider the case of an isotropic conductor with forces and fluxes given by (14), so that

$$
\begin{aligned}
& -\nabla \phi_{\mathrm{e}}=\mathbf{y}_{\mathrm{e}}-c \hat{\mathbf{j}}_{\mathrm{e}}+N_{\mathrm{e} S} \hat{\mathbf{j}}_{S}+R_{\mathrm{ee}} \mathbf{j}_{\mathrm{e}}+R_{\mathrm{e} S} \mathbf{j}_{S}, \quad \text { and } \\
& -\nabla \theta=\mathbf{y}_{S}-c \hat{\mathbf{j}}_{S}-N_{\mathrm{e} S} \hat{\mathbf{j}}_{\mathrm{e}}+R_{\mathrm{e} S} \mathbf{j}_{\mathrm{e}}+R_{S S} \mathbf{j}_{S}, \quad \text { where } \\
& |\underline{J}|^{2}=a_{\mathrm{e}}\left|\mathbf{j}_{\mathrm{e}}\right|^{2}+a_{S}\left|\mathbf{j}_{S}\right|^{2}, \quad c=\left(a_{\mathrm{e}} \mathbf{y}_{\mathrm{e}} \cdot \hat{\mathbf{j}}_{\mathrm{e}}+a_{S} \mathbf{y}_{S} \cdot \hat{\mathbf{j}}_{S}\right) /\left(a_{\mathrm{e}} \hat{\mathbf{j}}_{\mathrm{e}} \cdot \hat{\mathbf{j}}_{\mathrm{e}}+a_{S} \hat{\mathbf{j}}_{S} \cdot \hat{\mathbf{j}}_{S}\right),
\end{aligned}
$$

where $a_{\mathrm{e}}, a_{S}$ are positive scale factors having compatible physical dimensions, and $\mathbf{y}_{\mathrm{e}}, \mathbf{y}_{S}$ are arbitrary vectors. Although the contribution of the terms in $\mathbf{y}_{\mathrm{e}}, \mathbf{y}_{S}, N_{\mathrm{e}}$ to total dissipation is zero, the electromotive power is given by:

$$
-\mathbf{j}_{\mathrm{e}} \cdot \nabla \phi_{\mathrm{e}}=\left(\mathbf{y}_{\mathrm{e}}-c \hat{\mathbf{j}}_{\mathrm{e}}+N_{\mathrm{e} S} \hat{\mathbf{j}}_{S}\right) \cdot \mathbf{j}_{\mathrm{e}}+R_{\mathrm{ee}}\left|\mathbf{j}_{\mathrm{e}}\right|^{2}+R_{\mathrm{e} S} \mathbf{j}_{S} \cdot \mathbf{j}_{\mathrm{e}},
$$

involving terms that change sign whenever the signs of $\mathbf{j}_{\mathrm{e}}, \mathbf{j}_{S}$ are reversed and quadratic terms that do not. This is similar to the form proposed by Thomson [34], p. 130 (or [35], p. 317 ), becoming essentially the same whenever $\mathbf{j}_{S} \propto \mathbf{j}_{\mathrm{e}}$ and $N_{\mathrm{e} S}=0$.

In a more general setting, work-free forces or fluxes may be associated with constrained reversible coupling of various processes, a concept already familiar in biochemical energy transduction $[13,25]$. One can then make a distinction between processes that are tightly coupled, with minimal dissipation, and those that are loosely coupled with dominant dissipation. Whereas the Seebeck-Peltier effect most probably falls into the first category, the Thomson effect no doubt belongs in the second. As discussed in the Introduction, the classical thermoelectric analyses of Thomson and Boltzmann involve a combination of these effects, which tends to obscure this distinction.

\subsubsection{Non-dissipative Heat Flux}

The interesting question arises as the existence of materials with microstructure admitting equilibrium states in which only the heat flux is involved explicitly, such that

$$
\mathbf{q} \cdot \nabla \theta=0, \quad \text { with }|\mathbf{q}| \neq 0,|\nabla \theta| \neq 0
$$

To make this plausible, we apply Thomson's idea of a composite microstructure [34] to the strictly thermal heat-pump, ${ }^{16}$ involving steady heat exchange at respective rates $Q_{\mathrm{H}}>$ $0, Q_{\mathrm{C}}>0, Q_{\mathrm{A}}<0$, with heat reservoirs at high, low and ambient temperatures $\theta_{\mathrm{H}}, \theta_{\mathrm{C}}, \theta_{\mathrm{A}}$, where $\theta_{\mathrm{C}} \leq \theta_{\mathrm{A}} \leq \theta_{\mathrm{H}}$. The steady-state energy and entropy balances for the reversible version are:

$$
Q_{\mathrm{C}}+Q_{\mathrm{A}}+Q_{\mathrm{H}}=0, \quad \frac{Q_{\mathrm{C}}}{\theta_{\mathrm{C}}}+\frac{Q_{\mathrm{A}}}{\theta_{\mathrm{A}}}+\frac{Q_{\mathrm{H}}}{\theta_{\mathrm{H}}}=0 .
$$

\footnotetext{
${ }^{15}$ Lebon et al. [19] employ the classical designation "gyroscopic" [36] for such forces but, following Onsager, do not include them in their treatment of thermoelecticity.

${ }^{16}$ The basis for the absorption refrigerator (http://en.wikipedia.org/wiki/Einstein_refrigerator), with thermoelectric counterpart being more relevant to the present discussion.
} 
To make the point clear, let us assign a definite spatial orientation with Cartesian coordinates, according to $A, C, H \rightarrow x, y, z$, with $\theta_{\mathrm{A}}=\theta+\Delta_{x} \theta$, etc. Then, for a microscopically small device, ${ }^{17}$ the relations (40) give, to terms $O\left([\Delta \theta]^{2}\right)$,

$$
Q_{x} \Delta_{x} \theta+Q_{y} \Delta_{y} \theta+Q_{z} \Delta_{z} \theta=0
$$

the finite-difference analogue of (39). More generally, the relations (40) are special cases of $\nabla \cdot \mathbf{q}=0$ and $\nabla \cdot(\mathbf{q} / \theta)=0$, from which (39) follows. Thus, on scales much larger than the hypothetical heat-pump, the effect is to redirect an effective-continuum heat flux in such a way as to achieve orthogonality with the temperature gradient. This is reminiscent of related effects postulated by Thomson ([35], I-pp. $266 \mathrm{ff}$.) for crystalline conductors and would no doubt also require an anisotropic microstructure.

\section{Conclusions}

The foregoing analysis, based on contemporary thermodynamics, provides an alternative route to Thomson's classical formulae, along with an interpretation in terms of standard thermodynamic variables. From the vantage point of the present theory, various criticisms of Thomson's formulae may be seen to arise from an incomplete accounting for a reversible, rate-independent thermochemical coupling between heat and electrical flows.

The above coupling may be viewed as a special case of the thermodynamic orthogonality envisaged by Ziegler and Edelen, who allow for a fully non-linear model with a more general dissipative potential than the Rayleigh form assumed by Onsager. The theory also admits the possibility of non-dissipative heat conduction which the present analysis suggests might be realized by the appropriate thermoelectric microstructure.

Finally, the preceding analysis also provides a rudimentary approach to thermoelectric effects in elastic conductors, but there remain numerous questions as to the proper treatment of superficial charge concentration. As a test of the present predictions, there is an obvious need for comparison with experiments connecting the effects of stress on Seebeck potential and Peltier heat, which would take us well beyond the mainly theoretical concerns of the present paper.

Open Access This article is distributed under the terms of the Creative Commons Attribution Noncommercial License which permits any noncommercial use, distribution, and reproduction in any medium, provided the original author(s) and source are credited.

\section{References}

1. Boltzmann, L.: Zur Theorie der thermoelektrischen Ersheinungen. Sitzungber. Akad. Wiss. Wien, Abt II 96, 1258-1297 (1887)

2. Bridgman, P.W.: Thermo-electromotive force, Peltier heat, and Thomson heat under pressure. Proc. Am. Acad. Arts Sci. 53(4), 269-386 (1918)

3. Bridgman, P.W.: A critical thermodynamic discussion of the Volta, thermo-electric and thermionic effects. Phys. Rev. 14(4), 306 (1919)

4. Bridgman, P.W.: On the application of thermodynamics to the thermo-electric circuit. Proc. Natl. Acad. Sci. 15(10), 765-768 (1929)

\footnotetext{
${ }^{17}$ E.g. a nanoscopic thermoelectric circuit with heterojunctions properly arranged with respect to the given
} coordinate axes. 
5. Burak, Y.I., Galapats, B.P.: Thermodynamic fundamentals and investigation of surface phenomena in electrically conducting bodies. Mater. Sci. 17(5), 436-442 (1982)

6. Callen, H.B.: The application of Onsager's reciprocal relations to thermoelectric, thermomagnetic, and galvanomagnetic effects. Phys. Rev. 73(11), 1349 (1948)

7. Callen, H.B.: Thermodynamics and an Introduction to Thermostatistics. Wiley, New York (1985)

8. Carlson, D.E.: Linear thermoelasticity. In: Truesdell, C. (ed.) Handbuch der Physik, vol. VIa/2. Springer, Berlin (1972)

9. Clausius, R.: Die Mechanische Wärmetheorie-Band 2. Die Mechanische Behandlung der Electricität, vol. 2/2. Friedrich Vieweg und Sohn, Braunschweig (1879). Digital copy available at: http://gallica. bnf.fr and http://books.google.com/books

10. Coleman, B.D.: Thermodynamics of materials with memory. Arch. Ration. Mech. Anal. 17(1), 1-46 (1964)

11. Coleman, B.D., Dill, E.: On the thermodynamics of electromagnetic fields in materials with memory. Arch. Ration. Mech. Anal. 41, 132-162 (1971)

12. Edelen, D.G.: Primitive thermodynamics: a new look at the Clausius-Duhem inequality. Int. J. Eng. Sci. 12(2), 121-141 (1974)

13. Goddard, J.D.: A fundamental model for carrier-mediated energy transduction in membranes. J. Phys. Chem. 89(9), 1825-1830 (1985)

14. Goddard, J.D.: On material velocities and non-locality in the thermo-mechanics of continua. Int. J. Eng. Sci. 48(11), 1279-1288 (2010)

15. Gurtin, M.E., Vargas, A.S.: On the classical theory of reacting fluid mixtures. Arch. Ration. Mech. Anal. 43(3), 179-197 (1971)

16. Hall, E.H.: Inferences from the hypothesis of dual electric conduction; the Thomson effect. Proc. Natl. Acad. Sci. 6(3), 139-154 (1920)

17. Hall, E.H.: The Thomson effect and thermal conduction in metals. Proc. Natl. Acad. Sci. 6(10), 613-621 (1920)

18. Kittel, C.: Introduction to Solid State Physics. Wiley, New York (1986)

19. Lebon, G., Jou, D., Casas-Vázquez, J.: Understanding Non-equilibrium Thermodynamics. Springer, Berlin (2008)

20. Lewis, G.N., Randall, M.: Thermodynamics and the Free Energy of Chemical Substances. McGraw-Hill, New York (1923)

21. Lewis, G.N., Eastman, E.D., Rodebush, W.H.: The heat capacity of electro positive metals and the thermal energy of free electrons. Proc. Natl. Acad. Sci. 4(2), 25-29 (1918)

22. Maugin, G.A.: The Thermomechanics of Nonlinear Irreversible Behaviors: An Introduction. Nonlinear Science, vol. 27. World Scientific, Singapore (1999)

23. Miller, D.G.: Thermodynamics of irreversible processes. The experimental verification of the Onsager reciprocal relations. Chem. Rev. 60(1), 15-37 (1960)

24. Miner, A., Majumdar, A., Ghoshal, U.: Thermoelectromechanical refrigeration based on transient thermoelectric effects. Appl. Phys. Lett. 75(8), 1176-1178 (1999)

25. Mitchell, P.: Chemiosmotic coupling in energy transduction: A logical development of biochemical knowledge. J. Bioenerg. Biomembr. 3(1), 5-24 (1972)

26. Mott, N.F.: The Theory of the Properties of Metals and Alloys. Dover, New York (1958)

27. Müller, I.: A thermodynamic theory of mixtures of fluids. Arch. Ration. Mech. Anal. 28(1), 1-39 (1968)

28. Nye, J.F.: Physical Properties of Crystals: Their Representation by Tensors and Matrices. Oxford Science Publications. Clarendon, Oxford (1957)

29. Onsager, L.: Reciprocal relations in irreversible processes. I. Phys. Rev. 37(4), 405 (1931)

30. Sato, N., et al.: Novel MEMS power generator with integrated thermoelectric and vibrational devices. In: 13th Ann. Conf. Solid-State Sensors, Actuators and Microsystems, vol. 1, pp. 295-298 (2005)

31. Sommerfeld, A.: Lectures on Theoretical Physics-Thermodynamics and Statistical Mechanics, vol. V. Academic Press, New York (1964)

32. Thomson, W.: On a mechanical theory of thermo-electric currents. Proc. R. Soc. Edinb., 91-98 (1851), see also, Thomson (1882), Vol. I, pp. 237-266

33. Thomson, W.: Account of researches in thermo-electricity. Proc. R. Soc. Lond. 7, 49-58 (1854a), see also, Thomson (1882), Vol. I, pp. 460-468

34. Thomson, W.: On the dynamical theory of heat. Part V. Thermo-electric currents. Trans. R. Soc. Edinb. 7, 123-171 (1854b), see also, Thomson (1882), Vol. I, pp. 232-291

35. Thomson, W.: (various titles). In: Mathematical and Physical Papers, vol. I-II of 3, Cambridge University Press, Cambridge, UK, pp. I-232-91, I-316-25, I-460-68, II-173-74 (1882) (digital copy available at: http://gallica.bnf.fr)

36. Ziegler, H.: Proof of an orthogonality principle in irreversible thermodynamics. Z. Angew. Math. Phys. 21(6), 853-883 (1970) 